\title{
Regulatable systems: applications in gene therapy and replicating viruses
}

\author{
Siamak Agha-Mohammadi and Michael T. Lotze \\ University of Pittsburgh Medical Center, Pittsburgh, Pennsylvania, USA \\ Address correspondence to: Siamak Agha-Mohammadi, W1543 Biomedical Science Tower, \\ Lothrop Street, University of Pittsburgh Medical Center, Pittsburgh, Pennsylvania 15261, USA. \\ Phone: (412) 624-9375; Fax: (412) 624-1172; E-mail: mohammadis@msx.upmc.edu.
}

Perspective SERIES On cancer biotherapy

David H. Kirn, Editor

Effective gene-based therapies require efficient delivery of therapeutic genes to targeted mammalian cells as well as regulatable gene expression. Advances in gene therapy have mainly focused on the development of viral systems - adenovirus, retrovirus, adeno-associated virus, herpes virus, autonomous parvoviruses - and nonviral systems, such as direct injection of naked or conjugated DNA, for delivering transgenes into mammalian cells. Most of the currently applied gene delivery systems rely on strong viral promoters to drive high levels of expression in a wide range of tissues, although promoters from the cellular genes for phosphoglycerate kinase, actin and histones, have also been used with varying degrees of success. These promoters exhibit constitutive expression of proteins, which can cause cellular toxicity and may select for the downregulation of the effector systems.

Recent approaches to gene therapy have emphasized the need for gene delivery vectors that can efficiently introduce and control expression of foreign genes in a dose-dependent and reversible manner. Indeed, in certain disorders, only a specific range or dose of the therapeutic protein will achieve a successful outcome (1-3).

In clinical applications, it will be beneficial to regulate the transgene expression in order to maintain protein concentrations within the therapeutic window and to optimize efficacy in response to the evolving nature of the disease. A regulatable system would be of value in modifying specific therapies if it could offer tight regulation in response to pharmacologic agents that can be safely and repetitively administered. For cancer gene therapy, therapeutic gene products that would be particularly valuable to control in this way include cytokines (see Agha-Mohammadi and Lotze, this Perspective Series, ref. 4), prodrug activating enzymes (see Springer and Niculescu-Duvaz, this Perspective Series, ref. 5), ribozymes, antibodies, tumoricidal genes, or antisense oligonucleotides. Regulatable systems also have great utility in controlling the expression of the vector delivering the therapeutic gene. If replicating viruses are to be used to deliver such genes or to lyse tumors directly, regulation of viral early promoters may be required to control the rate of viral replication and enhance the safety of the recombinant virus.

First-generation regulatable systems, based on naturally occurring inducible promoters, generally suffer from high basal expression of the utilized promoter, weak induction of transgene expression, and reliance on inducible agents that exert pleiotropic effects on mammalian cells. Chimeric regulatable systems, devised to overcome these limitations, incorporate var- ious prokaryotic and eukaryotic elements and offer greater specificity than can be achieved using natural inducible promoters. Transactivators in these chimeric systems are designed to interact specifically with sequences engineered into the vector. Recently developed chimeric systems are regulated by tetracycline (6), the progesterone antagonist RU486 (7), the insect hormone ecdysone (8), or rapamycin (FK506) (9). These drugs or hormones (or their analogs) act on modular transactivators composed of natural or mutant ligand binding domains and intrinsic or extrinsic DNA binding and transcriptional activation domains.

\section{Chimeric regulatable systems}

Four efficient chimeric regulatable systems have been developed within the past decade (Figure 1). The tetracycline-repressed regulatable system (TrRS) (6) is based on the tetracycline resistance operon of Escherichia coli. This system exploits the specificity of the tet repressor (tetR) for the tet operator sequence (tet $O$ ), the sensitivity of tetR to tetracycline, and the ubiquitous activity of the potent herpes simplex virus transactivator (VP16) in eukaryotic cells. The TrRS uses a conditionally active chimeric tetracycline-repressed transactivator (tTA) that was created by fusing the $\mathrm{COOH}$-terminal 127 amino acids of VP16 to the $\mathrm{COOH}$ terminus of the tetR protein. In the absence of tetracycline, the tetR moiety of tTA binds with high affinity and specificity to a tetracycline-regulated promoter (tRP), a regulatory region comprising seven repeats of tetO placed upstream of a minimal human cytomegalovirus (CMV) promoter. Once bound to the tRP, the VP16 moiety of tTA transactivates the target gene by promoting assembly of a transcriptional initiation complex. However, binding of tetracycline to tetR leads to a conformational change in tetR accompanied with loss of tetR affinity for tet $O$, allowing transgene expression to be silenced by administering tetracycline. Luciferase activity is regulated over a range of one to five orders of magnitude in response to tetracycline in selected clones that express tTA and the luciferase cDNA under control of the tRP. The tetracycline-regulatable system (TRS) is the most widely used regulatable system and its applications in gene therapy are emphasized in the discussion later here.

The progesterone regulatable system (PRS) also shows three to four orders of magnitude induction of transgene in response to RU486. In this system, the transactivator consists of a VP16 transactivation domain, the ligand-binding domain of a mutant human progesterone receptor and the yeast GAL4 DNA-binding domain. 


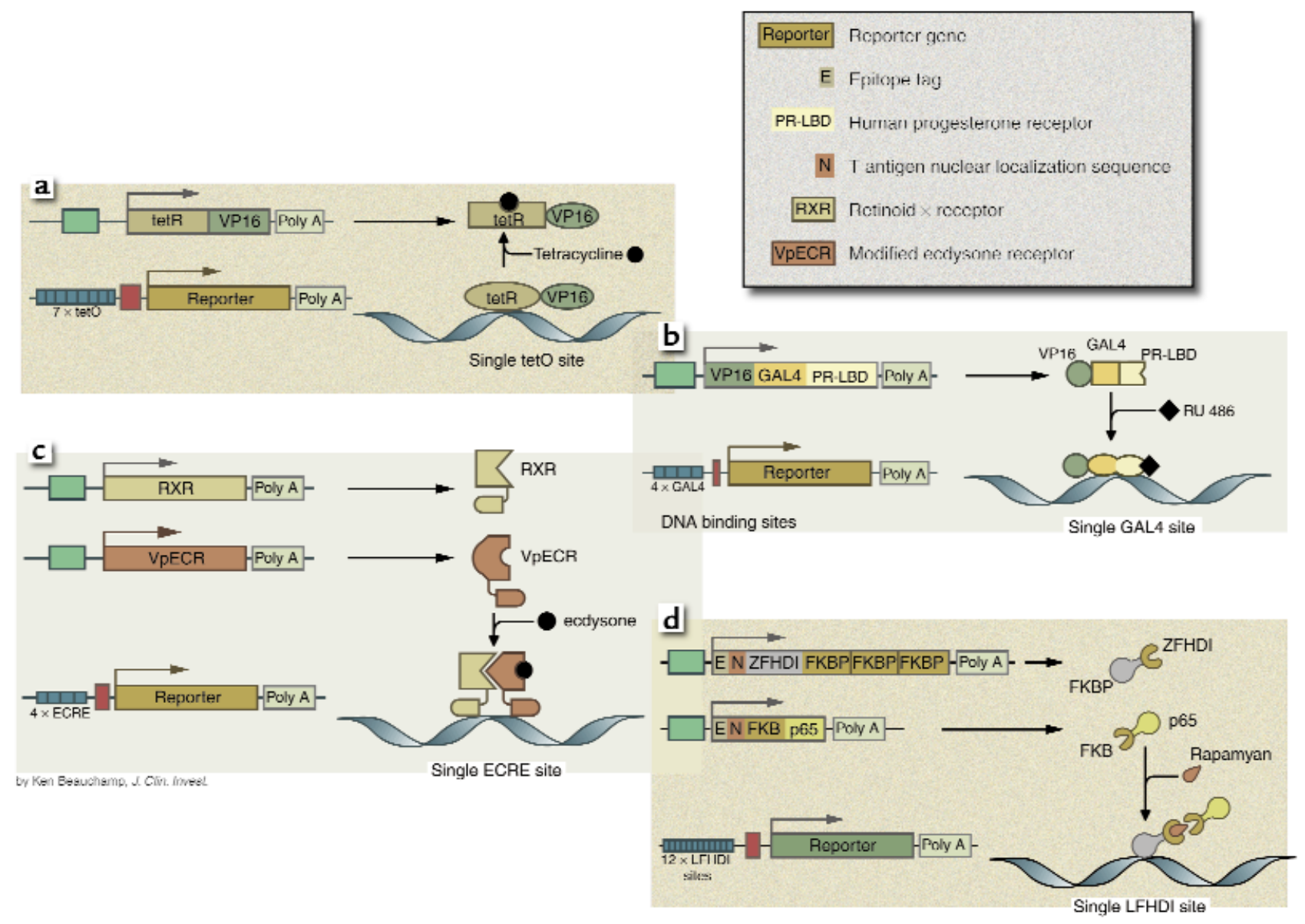

Figure 1

Chimeric regulatable systems have been designed to allow conditional expression of a reporter gene or a gene of therapeutic interest. In each of these systems, the transgene is transcriptionally silent until activated (or derepressed) by the addition (or removal) of a soluble hormone or drug. The drawing on the right side of each panel depicts the conditional binding of a transcription complex to one control element in an array of such elements (blue boxes) placed upstream of a minimal promoter or a TATA box (red boxes). (a) The tetracycline-regulatable system, the best studied of these systems, employs a chimeric, tetracycline-repressed transactivator (tTA; a fusion of tetR and truncated VP16 transactivator). In the absence of tetracycline, tTA binds with high affinity and specificity to a tetracycline-regulated promoter (tRP), a regulatory region comprising 7 repeats of tetO placed upstream of a minimal human $\mathrm{CMV}$ promoter. As described in the text, this system can be altered to allow for tetracycline-dependent, rather than tetracycline-repressible, transcription. (b) In the progesterone-regulatable system, the transactivator consists of VP16, the yeast GAL4 DNA-binding domain, and the ligand-binding domain of a mutant human progesterone receptor (PR-LBD). RU486 activates the transactivator by promoting the binding of the GAL4 DNA-binding domain to its consensus elements. (c) In the ecdysone-regulatable system, a heterodimeric complex of a modified ecdysone receptor component ( $\mathrm{VPECR}$ - a fusion of VP16, the $\mathrm{NH}_{2}$-terminal truncation of a mutant ecdysone receptor and the DNA-binding domain of the glucocorticoid receptor) and the retinoid X receptor ( $R X R)$ is inactive until stimulated by its ligand, an insect hormone foreign to human tissues. In the presence of ecdysone or its synthetic analog muristerone, this functional ecdysone receptor complex binds to 4 repeats of a unique response element (ECRE) in the target promoter. (d) The rapamycin-regulatable system uses rapamycin or its analog to bring together the functional units of a bipartite transcription factor. The bipartite transcription factor consists of two chimeric human peptides composed of ZFHD1 (the DNA binding unit) and FKBP12, and truncated cellular protein FRAP and truncated NF-kB p65 protein. In the presence of rapamycin, the ZFHD1-FKBP protein binds to the FRAP-p65 chimera to form a complex that activates 12 ZFHD1 sites in the ZFHD1-dependent promoter. E is an epitope tag and $\mathrm{N}$ is the SV40 T antigen nuclear localization sequence.

Endogenous hormones cannot induce the transactivator, but RU486 effectively activates the transactivator by promoting the binding of GAL4 DNA-binding domain to its consensus elements. Transgenic mice have been established that express the transactivator, as well as a human growth hormone (hGH) gene driven by 4 GAL4 DNA-binding sequences upstream of a TATA box. Only mice given RU486 express hGH, and the transgene can be induced rapidly, within 12 hours of RU486 administration. This system suffers from slow de-induction in vivo, taking up to 100 hours to reach uninduced basal expression (7). In mice expressing erythropoietin (Epo) under control of the PRS or TrRS, the limitation of the latter system becomes apparent: Hematocrit levels of mice could be conveniently up- and downregulated by doxycycline (Dox) but were hardly reversible on withdrawing RU486 administration. The slow de-induction of PRS is a consequence of the long half-life of RU486 and its poor diffusion within tissues (10). The PRS is also efficient as a self-contained unit, producing about 150fold regulation when delivered on a recombinant herpes simplex virus (11). Burcin et al. (12) recently developed a 
modified RU486-dependent transactivator in which the VP16 domain is replaced with a less immunogenic sequence, the $\mathrm{COOH}$-terminal 264 amino acids of the transcription factor NF-kB p 65 protein. Animal studies show that this system functions comparably to the PRS when delivered on a single recombinant adenovirus. However, RU-486, which has been used to induce elective abortions, has been at the center of political controversy in the United States, and this drug has not received approval by the Food and Drug Administration.

The ecdysone-regulated system (ERS) and the rapamycin-regulatable system (RRS) both require simultaneous expression of their three components. The RRS uses a "dimerizer" drug to bring together the functional units of a bipartite transcription factor. The bipartite transcription factor consists of two chimeric peptides composed of human proteins. These include a chimeric protein of ZFHD1 (DNA binding unit) and FKBP12 and a second chimeric protein of a truncated version of the cellular protein FRAP and the COOH-terminal 189 amino acids of NF-kB p 65 protein. In the presence of rapamycin, the ZFHD1-FKBP protein binds to the FRAP-p65 chimera to form a complex that activates 12 ZFHD1 sites in the ZFHD1-dependent promoter. In a selected clone expressing the bipartite transcription factor and the reporter unit, Rivera and colleagues (9) noted greater than 1,600-fold hGH expression in a doseresponsive manner by rapamycin. Reporter gene activity in the absence of rapamycin was indistinguishable from that of mock-transfected controls. When cells stably expressing the constituents of the system were introduced into nude mice, maximal ( 800-fold) induction occurred 16 hours after treatment with rapamycin. The immunosuppressive activity of rapamycin prohibits the use of this system in this form (9), but biologically inert rapamycin analogs may be developed that overcome this limitation of the RRS. Recently, the RRS has been further evaluated for gene therapy applications. Magari et al. (13) reexamined the regulation of the hGH in mice receiving cells that stably expressed the components of the system, and they reported efficient and dose-dependent upregulation of hGH within 24-48 hours of oral or intravenous administration of rapamycin. However, hGH remained above the basal levels for up to 12 days after a single injection of rapamycin. The authors argue that the prolonged kinetics of hGH results from the slow degradation of hGH mRNA molecules (13). Nevertheless, lack of complete de-induction of the system due to the presence of intracellular rapamycin cannot be ruled out. Delivery of the system by two adeno-associated virus vectors (in 1:1 ratio), one expressing the 2 units of the transcription factor chimeras and the second containing the Epo gene under the ZFHD1-dependent promoter, has also produced promising results in immune-competent mice. Administration of rapamycin resulted in a slow 200fold induction of plasma Epo with prolonged deinduction of the system to basal expression. Stable expression of Epo was achieved for 6 months in mice and for at least 3 months in a rhesus monkey (14).
The ERS is based on the insect hormone ecdysone and its functional receptor. The natural functional ecdysone receptor is a heterodimeric complex of the ecdysone receptor and the product of the ultraspiracle gene (USP). The mammalian ERS consists of a modified ecdysone receptor component (containing a fusion between VP16 and the $\mathrm{NH}_{2}$-terminal truncation of a mutant ecdysone receptor with DNA-binding specificity of the glucocorticoid receptor) and the mammalian homologue of USP, the retinoid X receptor. In the presence of ecdysone or its synthetic analog muristerone, this functional ecdysone receptor complex binds to four repeats of a unique response element in the target promoter. Stable cells expressing the modified ecdysone receptor, the retinoid $\mathrm{X}$ receptor and the reporter construct displayed approximately 20,000-fold induction of reporter gene activity. Furthermore, reporter gene induction could be controlled quantitatively over a wide range by altering the concentration of the inducing agent. The ERS is more than tenfold more efficient than the TRS in comparative studies (8) but has received less attention, in part because it requires simultaneous expression of its three components, and in part because the effects of ecdysone in the human body are unknown.

\section{Advantages of the TRS}

The TRS offers many advantages, based on the characteristics of tetR-tet $O$ binding and the properties of the tetracycline family of drugs, which make it particularly attractive for regulation of gene expression in vitro and in vivo. Since its introduction about 7 years ago, the TRS has been extensively used to study gene expression in stable cell lines and transgenic animals (S. Agha-Mohammadi, submitted for publication). Unlike the ERS or RRS, which require three components, the 2 units of the TrRS can be conveniently delivered on a single vector without compromising its efficiency (15). Furthermore, the pharmacokinetics, pharmacodynamics, and side effects of tetracycline and its derivatives are well known. Unlike other activators, such as steroids or rapamycin that have broad spectrum of activity in eukaryotic cells, tetracycline's high affinity for the tet $R$ enables the use of this antibiotic at concentrations that cause little adverse effects on mammalian cells and transgenic animals. In addition, certain tetracycline analogs, such as Dox, crosses placental barrier and are also secreted into milk, making this system particularly suitable for transgenic work. The TrRS is also distinguished from the other regulatable systems by its impressive degree of regulation and the ability to alter the kinetics of the response using different tetracycline analogs (16). Moreover, the system can be switched off and then switched on within 72 hours when using oxytetracycline (16). A final, unique advantage lies in the fact that, whereas all other chimeric systems either induce or suppress transgene expression, the tetracycline system can be engineered to work in either direction or in both ways simultaneously (17-19). Recently, a combination of both downregulatory and upregulatory variants of the tetracycline-regulatable system were 
developed and used to achieve exclusive control of two gene activities within the same cells at different concentrations of Dox (20).

\section{Tetracycline-induced regulatable system}

The original TrRS functions as a downregulatory system in the presence of tetracycline and is useful if gene expression is to be maintained in a switched-on state for long periods. A tetracycline-induced regulatable system (TiRS), on the other hand, may be preferred if gene expression is to be sustained in a switched-off state for long periods, if long-term exposure to tetracyclines is undesirable, or if rapid gene induction is required. Several permutations of the original system have been described that meet these requirements. The Tet-on system uses a reverse tTA (rtTA) is used that displays opposite binding properties compared with the wild-type tetR and thus requires tetracycline for binding to tet $O$. The rtTA has four amino acid exchanges in tetR, three of which have been implicated in the conformational change of tetR during induction. As with the TrRS, the Tet-on system responds differently to various tetracycline analogs. Dox and anhydrotetracycline are the most efficient tetracycline analogs in activating the Tet-on system, producing over a 1,000-fold dose-dependent induction in a selected clone expressing the rtTA and a reporter gene. The Tet-on system shows rapid kinetics of upregulation and thus overcomes the delay in TrRS activity after removal of tetracycline (17). Another TiRS (18) uses a fusion protein between tetR and the KRAB repressor domain of the human Kox1 zinc finger protein (tetR$\mathrm{KRAB}$ ) to achieve tetracycline-relieved silencing of eukaryotic promoters. In the absence of tetracycline, tetR-KRAB exerts its effect by binding to seven tet $O$ sequences built upstream of an intact CMV IE promoter. In a selected clone expressing tetR-KRAB, up to 50-fold induction occurred after 3 days of tetracycline treatment.

An alternative approach is based on interference with formation of transcriptional initiation complex by tTA. In transient expression studies (19), a vector containing tet $O$ sequences flanking a TATA box of a modified viral promoter showed maximal repression in response to tTA but not tetR. Infection of a high tTAexpressing cell line with a recombinant human CMV carrying this plasmid resulted in 60- to 120 -fold tTAmediated gene repression that was relieved by tetracycline (19). An extension of this approach uses tetR, instead of tTA, to interfere with CMV IE promoter activity by positioning two tetO sites downstream of the TATA element. Using this tetR-mediated transcription repression switch in in vivo models, Yao et al. (21) achieved approximately 60-fold tetR-mediated regulation and showed that transgene silencing could be reversed when tetracycline was administered.

\section{Targeting the TRS}

The success of a therapeutic strategy involving gene therapy ultimately depends on its specificity, which can be achieved through use of targeted vectors or targeted transcriptional expression. As there are few vectors, if any, that allow efficient targeted transduction, most investigators have relied on the latter approach. Alongside such spatial transcriptional targeting, it will often be advantageous to regulate the timing of transgene expression. To establish targeted and regulated gene expression, two strategies have been followed. The first approach uses tissue-specific enhancers/promoters to express tTA after direct plasmid delivery, in transgenic animals, or after viral delivery to target expression and regulation of a transgene (S. Agha-Mohammadi, submitted for publication).

In the second approach, tet $O$ sites are integrated into an existing tissue-specific enhancer/promoter. Rose and MacDonald (22) replaced in turn three functional elements of rat Elastase I enhancer with a tet $O$ element to create an enhancer that was inactive in the absence of tTA but could be activated two- to eightfold by tTA, relative to the unmodified enhancer. tTA-activated tet $O$ substituted enhancers produced tetracycline-dependent regulation ranging from 190 - to 330 -fold in a pancreatic cell line. Depending on the substituted element and the cell line examined, little or no activity of the tTAactivated enhancers was observed in nonpancreatic cell lines. These results indicated that a single tet $O$ element can be incorporated into an enhancer to create a tissuespecific regulatable transcriptional control.

\section{Applying TRSs in cancer gene therapy}

Because they allow for the controlled expression of therapeutic genes, regulatable systems, particularly the TRS, can address many safety concerns surrounding gene therapy and may eventually enjoy wide use. Although, TRS has been successfully codelivered via separate vectors (Table 1), for effective gene delivery, the regulatory system must ultimately be contained on a single vector. Recently, a number of self-contained plasmids have been constructed. These plasmids fall into three groups: The first group expresses tTA from a viral or tissue-specific promoter as well as the tRP-transgene cassette (23). The second group, bicistronic vectors, express a reporter gene from a tRP unit, which is followed by tTA expression from an internal ribosome entry site element placed distal to the reporter gene, or vice versa. In the absence of tetracycline, these vectors allow tTA to promote transcription of the transgene and its own gene in an autoregulatory fashion (24). The third group, positive feedback regulatory vectors, express both the tTA and a transgene from a modified bidirectional promoter. Positive feedback regulatory vectors also function on the basis of the autoregulatory principle (15). Generally, the self-contained vectors function well as plasmids and also in the context of adenoviruses, retroviruses, adeno-associated virus, and herpes simplex virus, and they also display regulation comparable to the two-component delivery vectors. Overall, these preliminary results confirm that the basal expression of tRP is similar whether plasmids or viral vectors are used. The copy number of the tetracycline-regulated expression cassette is the most significant factor determining the basal level of expression of the gene of interest (25).

Despite many improvements in vectorology, there are still few reports of therapeutic gene regulation in vivo. Bohl et al. (26) have used two retroviruses to transduce pri- 
mary myogenic cells with tRP-mouse Epo and rtTA, achieving greater than 200-fold induction of Epo protein in response to Dox in vitro. After transplantation of these cells into skeletal muscle of animals and treatment with Dox for 2-3 weeks, they noted an increase in hematocrit from about $50 \%$ to about $75 \%$ (26). In an analogous experiment using tTA (27), engraftment of the cells in syngeneic recipient mice led to an increase of the hematocrit and serum Epo levels. Animals that received tetracycline showed significantly lower values than untreated animals. Recently, Pitzer et al. (28) demonstrated the potential of this system for ex vivo cancer gene therapy. These authors cotransduced a melanoma cell line with two recombinant retroviruses expressing tTA or $\mathrm{tRP}$ driving the human IL2 gene, and they demonstrated in vitro regulation of this cytokine. Furthermore, TrRS regulated IL-2 expression correlated inversely with tumor size in vivo.

\section{The promise of regulatable expression for cancer biotherapy}

Viral vectors offer the most efficient vehicle to deliver therapeutic genes in to target cells. Most ongoing gene therapy protocols use replication-defective recombinant viral vectors derived from retroviruses, adenoviruses, or herpesviruses. However, the importance of replicationcompetent viruses has been highlighted with the development of the adenovirus-derivative ONYX-015, which specifically targets p53-defective tumor cells (29; see also Heise and Kirn, this Perspective Series, ref. 30).

Controlled production of recombinant viruses represents an emerging application of regulatable system that could be incorporated directly into ONYX-015 or analogous biotherapeutic agents. Regulatable systems can be used to control all aspects of viral life cycle. Rittner et al. (31) used tetracycline-relieved silencing of eukaryotic promoters by tetR-KRAB (18) to control the expression of the adenovirus $E 2 A$ gene. In the absence of tetracycline, these investigators find that E2A expression is strongly repressed, leading to a block in viral
DNA replication and late gene expression. Similarly, Caplen et al. (32) recently applied the Tet-on system to control generation of retroviral particles delivered as adeno-retroviral chimeric viruses. In their system, recombinant adenoviruses carrying the retroviral gag.pol genes under the control of tRP were cotransduced with adenoviruses carrying a retroviral vector with rtTA. In vitro, higher doses of adeno-retroviral viruses yielded up to a 2,000-fold induction of retroviral particles, although parallel in vivo studies were disappointing. Another tetracycline-regulated viral replication switch was described recently that uses the tetR-mediated transcription repression switch. Using this switch, replication of wild-type herpes simplex virus could be regulated effectively by controlling the expression of a dominant negative mutant of the origin of DNA replication-binding protein, a protein required for viral replication (33). This system is inconvenient, as the viral DNA, the gene for the mutant protein, and the tetR gene were delivered on separate plasmids. Also, the tetR expressing vector had to be transfected in significant excess to permit robust regulation of the transgene by tetracycline. This requirement may interfere with efficient gene delivery and increase the risk that tet $\mathrm{R}$ will provoke an immune response. To address these concerns, we have developed a second-generation tetracycline-controlled positive feedback regulatory vector that demonstrates more than a 1,000-fold gene regulation from a single plasmid. This vector should be of value in modifying the life cycle of a recombinant viral vector (S. AghaMohammadi et al., manuscript in preparation).

Many of the current cancer gene therapy protocols use immunomodulatory cytokines such as IL-2, -4, 12 , and -18; GM-CSF; and TNF- $\alpha$. Cytokine gene delivery via replication-efficient adenoviruses is expected to enhance the elicited immune response against cancer cells (see Agha-Mohammadi and Lotze, this Perspective Series, ref. 4). For cytokine gene therapy using replication-efficient viruses, regulatable gene expres-

Table 1

Delivery of the components of TRS on two separate vectors or single self-contained vectors

TRS components on two separate vectors

Direct plasmid coinjection 1:1

Direct Plasmid co-injection 1:1

2 adenoviruses

2 adenoviruses

2 adenoviruses

Ex vivo gene therapy

TRS components on a single self-contained vector

Plasmid transfection

Bicistronic plasmid injection

Positive feedback regulatory plasmid

Retrovirus transduction

Retrovirus transduction

Retrovirus transduction

HSV injection into CNS

AAV injection into CNS

Adenovirus
tTA and tRP-luciferase

tTA and tRP-Luciferase

tTA and tRP- $\beta$-gal

tTA and tRP-EGFP

rtTA and tRP-EGFP

NLS-rtTA and tRP-EGFP

NLS-tTA and tRP- $\beta$-gal

rtTA and tRP-mouse Epo

tTA and tRP-p53

tRP-luciferase-IRIS-tTA

tTA-bitRP-mGMCSF

tTA and tRP-luciferase

tTA and tRP-thymidine kinase

tTA and tRP-IL $1 \beta$-converting enzyme

tTA and tRP-luciferase

tRP- $t T A$ and $t R P$-EGF

tTA and tRP-TNF- $\alpha$
50 -fold regulation in vivo

100 -fold regulation in vivo

190 -fold regulation in vitro

330 -fold regulation in vitro

119 -fold regulation in vitro

7 -fold regulation in vitro

80 -fold regulation in vitro

200 -fold regulation in vivo

85 -fold regulation in vitro 40 -fold regulation in vivo 50 to 300 -fold regulation in vitro

24- to 336 -fold regulation in vitro

350 -fold regulation in vitro

40 -fold regulation in vivo

7 -fold regulation in vivo

15 -fold regulation in vivo

Significant control in vitro 
sion offers the potential to coordinate the replication of the virus with the desired dose of the therapeutic gene product. The ability of replication-competent viruses to replicate and transduce many of the target cancer cells will ultimately generate very high cytokine expression. This is neither physiological nor without adverse effects, especially in the case of cytokines like IL-2, IL-12, and TNF- $\alpha$, which display high local and systemic toxicity when expressed at high doses from recombinant adenoviruses. Using regulatable systems, it would be possible to modify the expression of these cytokines to achieve the optimal therapeutic dose throughout the replication and transduction cycles. It may also be possible to activate the expression of the cytokine after an initial cycle of replication and transduction. Regulatable systems also provide the means to terminate the expression of a cytokine should adverse effects appear or safety concerns arise.

\section{Considerations for the future}

The real impact of regulatable promoters in gene therapy remains to be seen. The results so far demonstrate the potential of the TRS for regulating therapeutic gene levels, independent of the means of gene delivery and the vector chosen. Most investigators report a 10to 100 -fold transgene regulation when using the system in vitro and in vivo. This variability reflects the many poorly controllable aspects of these systems, including the cell type, the surrounding context of vector/integration site, the intracellular concentration of the transactivator, and the basal intracellular concentration of transgene.

For effective gene delivery, all the components of a regulatory system must be contained on a single vector. Furthermore, this vector must permit high expression of transgene on induction and low basal expression when repressed. So far, most of the reported vectors have shown relatively high basal expression and therefore limited efficiency of the system. Recently, we described a novel tetracycline-controlled positive feedback regulatory vector that transcribes both tTA and $\mathrm{mGM}$-CSF from a modified tTA-responsive bidirectional promoter. This vector demonstrated gene regulation in several cell lines ranging from 50- to 300fold. To our knowledge, this degree of regulation is the highest that has been described using the TRS on a single self-contained plasmid in transient studies (15). Still more efficient regulatory system will be necessary to achieve regulated gene expression in vivo. Consequently, we have recently developed a second-generation tetracycline-controlled positive feedback regulatory vector that demonstrates about a 1,000-fold gene regulation from a single plasmid. We are currently exploring this vector and studying the importance of cytokine expression level in cancer gene therapy (S. Agha-Mohammadi et al., manuscript in preparation).

1. Dranoff, G., et al. 1993. Vaccination with irradiated tumor cells engineered to secrete murine granulocyte-macrophage colony-stimulating factor stimulates potent, specific, and long lasting anti-tumor immunity. Proc. Natl. Acad. Sci. USA. 90:3539-3543.

2. Schmidt, W., et al. 1995. Cancer vaccines: the interleukin 2 dosage effect. Proc. Natl. Acad. Sci. USA. 92:4711-4714.
3. Naffakh, N., and Danos, O. 1996. Gene transfer for erythropoiesis enhancement. Mol. Med. Today. 2:343-348.

4. Agha-Mohammadi, S., and Lotze, M.T. 2000. Immunomodulation of cancer: potential use of selectively replicating agents. J. Clin. Invest. 105:1173-1176.

5. Springer, C.J., and Niculescu-Duvaz, I. 2000. Prodrug-activating systems in suicide gene therapy. J. Clin. Invest. 105:1161-1167.

6. Gossen, M., and Bujard, H. 1992. Tight control of gene expression in mammalian cells by tetracycline-responsive promoters. Proc. Natl. Acad. Sci. USA. 89:5547-5551.

7. Wang, Y., DeMayo, F.J., Tsai, S.Y., and O’Malley, B.W. 1996. Ligandinducible and liver-specific target gene expression in transgenic mice. Nat. Biotechnol. 15:239-243.

8. No, D., Yao, T.-P., and Evans, R.M. 1996. Ecdysone-inducible gene expression in mammalian cells and transgenic mice. Proc. Natl. Acad. Sci. USA. 93:3346-3351.

9. Rivera, V.M., et al. 1996. A humanized system for pharmacologic control of gene expression. Nat. Med. 2:1028-1032.

10. Serguera, C., Bohl, D., Rolland, E., Prevost, P., and Heard, J.M. 1999. Control of erythropoietin secretion by doxycycline or mifepristone in mice bearing polymer-encapsulated engineered cells. Hum. Gene Ther. 10:375-383.

11. Oligino, T., et al. 1998. Drug inducible transgene expression in brain using a herpes simplex virus vector. Gene Ther. 5:491-496.

12. Burcin, M.M., Schiedner, G., Kochanek, S., Tsai, S.Y., and O’Malley, B.W. 1999. Adenovirus-mediated regulable target gene expression in vivo. Proc. Natl. Acad. Sci. USA. 96:355-360.

13. Magari, S.R., Rivera, V.M., Iuliucci, J.D., Gilman, M., and Cerasoli, F. 1997. Pharmacologic control of a humanized gene therapy system implanted into nude mice. J. Clin. Invest. 100:2865-2872.

14. Ye, X., et al. 1999. Regulated delivery of therapeutic proteins after in vivo somatic cell gene transfer. Science. 283:88-91.

15. Agha-Mohammadi, S., and Hawkins, R.E. 1998. Efficient transgene regulation from a single tetracycline-controlled positive feedback regulatory system. Gene Ther. 5:76-84.

16. Agha-Mohammadi, S., Alvarez-Vallina, L., Ashworth, L.J., and Hawkins, R.E. 1997. Delay in resumption of the activity of tetracycline-regulatable promoter following removal of tetracycline analogues. Gene Ther. 4:993-997.

17. Gossen, M., et al. 1995. Transcriptional activation of tetracyclines in mammalian cells. Science. 268:1766-1769.

18. Deuschle, U., Meyer, W.K.H., and Thiesen, H.J. 1995. Tetracyclinereversible silencing of eukaryotic promoters. Mol. Cell. Biol. 15:1907-1914.

19. Kim, H.J., Gatz, C., Hillen, W., and Jones, T.R. 1995. Tetracycline repressor-regulated gene repression in recombinant human cytomegalovirus. J. Virol. 69:2565-2573.

20. Baron, U., et al. 1999. Generation of conditional mutants in higher eukaryotes by switching between the expression of two genes. Proc. Natl. Acad. Sci. USA. 96:1013-1018.

21. Yao, F., et al. 1998. Tetracycline repressor, tetR, rather than the tetRmammalian cell transcription factor fusion derivatives, regulates inducible gene expression in mammalian cells. Hum. Gene Ther. 9:1939-1950.

22. Rose, S.D., and MacDonald, R.J. 1997. Integration of tetracycline regulation into a cell-specific transcriptional enhancer. J. Biol. Chem. 272:4735-4739.

23. O'Brien, K., Otto, K., and Rao, R.N. 1997. Construction and characterization of a one-plasmid system for the controlled expression of genes in mammalian cells by tetracycline. Gene. 184:115-120.

24. Liang, X., Hartikka, J., Sukhu, L., Manthorpe, M., and Hobart, P. 1996. Novel, high expressing and antibiotic-controlled plasmid vectors designed for use in gene therapy. Gene Ther. 3:350-356.

25. Neering, S.J., Hardy, S.F., Minamoto, D., Spratt, S.K., and Jordan, C.T. 1996. Transduction of primitive human hematopoietic cells with recombinant adenovirus vectors. Blood. 88:1147-1155.

26. Bohl, D., Naffakh, N., and Heard, J.M. 1997. Long-term control of erythropoietin secretion by doxycycline in mice transplanted with engineered primary myoblasts. Nat. Med. 3:299-305.

27. Bohl, D., and Heard, J.M. 1997. Modulation of erythropoietin delivery from engineered muscles in mice. Hum. Gene Ther. 8:195-204.

28. Pitzer, C., Schindowski, K., Pomer, S., Wirth, T., and Zoller, M. 1999. In vivo manipulation of interleukin-2 expression by a retroviral tetracycline tet)-regulated system. Cancer Gene Ther. 6:139-146.

29. Heise, C.C., Williams, A.M., Xue, S., Propst, M., and Kirn, D.H. 1999. Intravenous administration of ONYX-015, a selectively replicating adenovirus, induces antitumoral efficacy. Cancer Res. 59:2623-2628.

30. Heise, C., and Kirn, D.H. 2000. Replication-selective adenoviruses as oncolytic agents. J. Clin. Invest. 105:847-851.

31. Rittner, K., Schultz, H., Pavirani, A., and Mehtali, M. 1997. Conditional repression of the E2 transcription unit in E1-E3-deleted adenovirus vectors is correlated with a strong reduction in viral DNA replication and late gene expression in vitro. J. Virol. 71:3307-3311. 
32. Caplen, N.J., et al. 1999. Adeno-retroviral chimeric viruses as in vivo transducing agents. Gene Ther. 6:454-459.

33. Yao, F., and Eriksson, E. 1999. A novel tetracycline-inducible viral replication switch. Hum. Gene Ther. 10:419-427.

34. Fishman, G.I., Kaplan, M.L., and Buttrick, P.M. 1994. Tetracycline-regulated cardiac gene expression in vivo. J. Clin. Invest. 93:1864-1868.

35. Dhawan, J., Rando, T.A., Elson, S.L., Bujard, H., and Blau, H.M. 1995. Tetracycline-regulated gene expression following direct gene transfer into mouse skeletal muscle. Somat. Cell Mol. Genet. 21:233-240.

36. Harding, T.C., Geddes, B.J., Noel, J.D., Murphy, D., and Uney, J.B. 1997. Tetracycline-regulated transgene expression in hippocampal neurones following transfection with adenoviral vectors. J. Neurochem. 69:2620-2623.

37. Yoshida, Y., and Hamada, H. 1997. Adenovirus-mediated inducible gene expression through tetracycline-controllable transactivator with nuclear localization signal. Biochem. Biophys. Res. Commun. 230:426-430.

38. Paulus, W., Baur, I., Boyce, F.M., Breakefield, X.O., and Reeves, S.A. 1996. Self-contained, tetracycline-regulated retroviral vector system for gene delivery to mammalian cells. J. Virol. 70:62-67.
39. Hwang, J.J., Scuric, Z., and Anderson, W.F. 1996. Novel retroviral vector transferring a suicide gene and a selectable marker gene with enhanced gene expression by using a tetracycline-responsive expression system. $J$. Virol. 70:8138-8141.

40. Yu, J.S., Sena-Esteves, M., Paulus, W., Breakefield, X.O., and Reeves, S.A. 1996. Retroviral delivery and tetracycline-dependent expression of IL1 beta-converting enzyme (ICE) in a rat glioma model provides controlled induction of apoptotic death in tumor cells. Cancer Res. 56:5423-5427.

41. Haberman, R.P., McCown, T.J., and Samulski, R.J. 1998. Inducible longterm gene expression in brain with adeno-associated virus gene transfer. Gene Ther. 5:1604-1611.

42. Ho, D.Y., McLaughlin, J.R., and Sapolsky, R.M. 1996. Inducible gene expression from defective herpes simplex virus vectors using the tetracycline-responsive promoter system. Brain Res. Mol. Brain Res. 41:200-209.

43. Hu, S.X., Ji, W., Zhou, Y., Logothetis, C., and Xu, H.J. 1997. Development of an adenovirus vector with tetracycline-regulatable human tumor necrosis factor alpha gene expression. Cancer Res. 57:3339-3343. 